\title{
TERRITOIRE MULTISITUÉ
}

\author{
Geneviève Cortes et Denis Pesche
}

Belin | L'Espace géographique

\section{3/4 - Tome 42 \\ pages 289 à 292}

\section{ISSN 0046-2497}

Article disponible en ligne à l'adresse:

http://www.cairn.info/revue-espace-geographique-2013-4-page-289.htm

Pour citer cet article :

Cortes Geneviève et Pesche Denis, « Territoire multisitué »,

L'Espace géographique, 2013/4 Tome 42, p. 289-292.

Distribution électronique Cairn.info pour Belin.

(c) Belin. Tous droits réservés pour tous pays.

La reproduction ou représentation de cet article, notamment par photocopie, n'est autorisée que dans les limites des conditions générales d'utilisation du site ou, le cas échéant, des conditions générales de la licence souscrite par votre établissement. Toute autre reproduction ou représentation, en tout ou partie, sous quelque forme et de quelque manière que ce soit, est interdite sauf accord préalable et écrit de l'éditeur, en dehors des cas prévus par la législation en vigueur en France. II est précisé que son stockage dans une base de données est également interdit. 


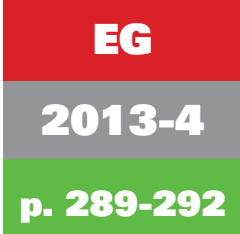

\section{Territoires multisitués}

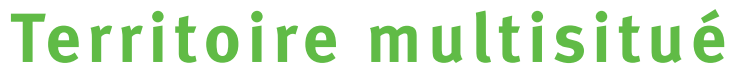

D

ans le champ des sciences sociales, saisir la multiplicité et la pluralité devient incontournable au regard de la complexité qui s'impose à nous. Symptomatique de cette quête de la complexité s'avère la déclinaison de diverses figures de la territorialité: supraterritorialité, interterritorialité, transterritorialité, extraterritorialité, multiterritorialité (Baudin et al., 2009). Y a-t-il encore la place pour une notion de plus, celle de "territoire multisitué »? Quel(s) sens lui donner? Quelle en est la portée heuristique? ${ }^{1}$

L'accroissement des mobilités et des dynamiques de réseaux stimule la conception et l'usage d'une notion a priori paradoxale telle que celle de territoire multisitué. À une vision classique du territoire - d'action, d'appropriation et de représentations d'un groupe social - qui serait celle d'un espace uni-localisé, contigu et délimité peut-on ajouter (ou lui opposer?) celle qui se réfèrerait à un espace de multilocalisation, de discontinuités, de dispersion et d'interactions entre lieux? Enchevêtrement des échelles, brouillage des limites, éclatement des espaces de référence et des ancrages marquent ces espaces archipels. Il s'agit en somme de savoir en quoi des dynamiques sociales, culturelles et économiques qui répondent à des logiques archipélagiques et réticulaires plus qu'aréolaires "font territoire ». Comment appréhender ces configurations territoriales d'un point de vue théorique, méthodologique et empirique? Enfin, comment les prendre en compte dans une réflexion plus large sur la gouvernance et le développement des sociétés et des territoires?

À notre connaissance, la notion de "territoire multisitué " en tant que telle, n'a pas véritablement été utilisée en géographie ou dans d'autres disciplines des sciences sociales. Cette notion prolonge les avancées de la réflexion géographique autour de plusieurs débats. Le premier renvoie au diagnostic de « fin des territoires » auquel on a pu rétorquer celui d'une complexification des configurations territoriales (Haesbert, 2001; Antheaume, Giraut, 2005; Vannier, 2009), ou encore la nécessité d'appréhender les nouvelles réalités des territoires circulatoires (Tarrius, 2005). Il s'agit donc de prendre en compte la polymorphie des relations socio-spatiales (Jessop et al., 2008) mais aussi et surtout de s'émanciper d'une conception par trop restrictive et traditionnelle du territoire régie par les principes de la fixité, de la souveraineté et de la continuité, de se plonger en quelque sorte dans " ces autres territoires " tels qu'évoqués par Guénola Capron et al. (2005). Indissociable de ce premier débat, le deuxième se réfère à la tension dualiste entre territoire et réseau qui a laissé place à une conception dialectique du territoire-réseau fondée sur l'articulation, voire l'enchevêtrement, de formations aréolaires et réticulaires. Il s'agit, en troisième lieu, d'emprunter la voie de Christine Romero lorsqu'elle parle de "territorialités discontinues " (2010) ou plus encore celle de Jean-Baptiste Arrault (2005) lorsqu'il propose de fonder la validité du concept de territoire-archipel sur « l'intersection ou la superposition de deux ordres de spatialité, un ordre continu et un ordre discontinu $"$ (p. 326).

Les antécédents relatifs à l'usage du terme "multisitué " ne sont pas totalement absents. Le terme de " ménage multisitué " par exemple (Pinedo-vasquez et al., 2009) fait référence à la dispersion résidentielle des membres du ménage comme dispositif réticulaire fonctionnel qui articule ville et campagne. Se référant tout autant au phénomène de dispersion, mais déplaçant l'unité sociale de référence, la notion de "village multisitué "

1. Ces questionnements ont fait l'objet d'un séminaire de recherche le 14 décembre 2010 organisé par l'unité ART-Dev, UMR 5281 de Montpellier. 
(Dia, 2010) renvoie aux réseaux sociaux migratoires qui se déploient depuis le village d'origine des migrants et qui permet, du même coup, la démultiplication et dissémination du site villageois dans d'autres lieux distants. Saskia Sassen, en se positionnant du point de vue sociopolitique et institutionnel, évoque l'existence de "territorialités multisituées " (multi-sited territoriality) dans un article sur ce qu'elle appelle les nouveaux assemblages des territoires liés à la globalisation (Sassen, 2008).

En tant que concept, il peut se définir comme un ensemble de lieux discontinus constitutif d'un espace fonctionnel et vécu (pratiques, activités, représentations), voire organisationnel dès lors que sont en jeu des processus d'action, de gestion et de coopération. Cependant, si la dispersion et la discontinuité sont constitutives des territoires multisitués, ils n'en sont pas des attributs uniques et suffisants. Car encore faut-il que cet espace soit structuré, organisé, construit sur la base d'un système relationnel qui lie socialement, économiquement, voire politiquement, plusieurs lieux dispersés et distants. Ces liens, précisément, empêchent l'éclatement, font en sorte que la fragmentation apparente continue à "faire » territoire. C'est aussi en ce sens que le territoire multisitué dépasse l'idée de localisation. La situation se distingue de la simple position dans l'espace ou localisation, en tant qu'elle est " une caractéristique géographique fondamentale d'un lieu, d'un espace, résultant de sa relation aux autres lieux ou espaces " (Brunet et al., 2009, p. 456). Une situation est donc toujours relative et changeante. Autrement dit, le territoire multisitué se construit, se lit, se décrypte à la façon d'un kaléidoscope, c'est à dire dans la pluralité et la variation de la situation relative et relationnelle de chacun des lieux et des réseaux qui le constituent. En s'inspirant des propos de François Laplantine dans son ouvrage Le Social et le Sensible (2005), on peut dire en somme que le territoire multisitué serait une construction " métisse "; loin de n'être qu'une simple juxtaposition d'éléments pluriels formant un tout, elle est un agencement d'éléments multiples qui donne des formes et des compositions différentes, des modulations et des variations. Ces agencements constituent en quelque sorte les différentes "pliures» du territoire multisitué. C'est précisément pour cette raison qu'il appelle également à un principe de méthode, celui proposé notamment par le courant de l'ethnographie multisituée (Marcus, 1995; Falzon, 2009) qui prône la démultiplication des observations d'un même objet en plusieurs sites.

Si le territoire multisitué est ici interrogé du point de vue de la géographie, il constitue une notion passerelle du fait de sa capacité à faciliter le dialogue avec d'autres disciplines. Du côté de la sociologie, cette notion fait écho aux travaux sur les recompositions des identités et des sociétés en lien avec les dynamiques de réseaux, ces derniers produisant de nouveaux agencements entre ressources, espaces et lieux, des ancrages multiples, dissociés, à caractère temporaire, de plus en plus fluides (Bauman, 1999; Kaufman, 2004). Du côté de la science politique, la notion de territoire multisitué renvoie à la question ardue des formes de contrôle des circulations et de la compatibilité entre mobilités et structures de pouvoir, ces dernières étant généralement ancrées sur un territoire donné, unifié. Comment évoluent les formes de l'action publique et les modes de gouvernance dans des configurations territoriales où l'on passe du schéma de la juxtaposition et de l'emboîtement des échelles (du local à l'international) à celui, plus complexe, de la superposition et de l'articulation d'espaces disjoints? La notion de multisituation évoque aussi celle de multipositionnalité des responsables politiques qui jouent de leurs ancrages multiples pour combiner des ressources de différentes natures dans des stratégies composites. En liant l'idée de multisituation à celle de territoire, on 
déplace la question de la multipositionnalité, attachée à un individu, pour envisager l'existence d'attachements croisés entre des lieux, au-delà des trajectoires spécifiques des individus qui contribuent à les relier.

Ces questionnements animent les contributions présentées dans ce numéro, qui s'interrogent sur la portée heuristique de la notion de territoire multisitué en la confrontant à plusieurs cas d'étude.

Dans sa contribution, Frédéric Giraut propose de positionner la notion de territoire multisitué par rapport à celles de postmodernité territoriale, d'une part, et de complexité territoriale, d'autre part. Il les teste à partir des configurations spatiales et des " ingénieries territoriales » de la fragmentation en Afrique du Sud post-apartheid et il explore ensuite la portée (et les limites) de la notion en questionnant sa conciliation possible avec les territorialités politiques et les espaces de la gouvernementalité.

Ce défi posé à la sphère du politique est également formulé par la contribution de Stéphanie Nasuti, Ludivine Eloy et François-Michel Le Tourneau. Celle-ci met à l'épreuve la notion au regard des stratégies d'usage des ressources par les populations quilombolas de l'Amazonie brésilienne. À partir d'une méthode d'analyse des systèmes de mobilités et de la dispersion résidentielle des familles, les auteurs mettent en évidence les dimensions identitaires et institutionnelles de leur espace de vie multipolaire, qui font de celui-ci un territoire partagé. Paradoxalement, la mobilité, la réticularité et la flexibilité territoriale apparaissent comme une condition même du contrôle et de la gestion de la ressource localisée.

Pour Stéphanie Lima, la multisituation territoriale nait également du fait mobilitaire et de la dispersion qui fait ressource pour les populations. En analysant les évolutions sur la longue durée des migrations internationales dans la région des Kayes au Mali, l'auteure décrypte la fabrique des territoires multisitués en plaçant au cœur de l'analyse le migrant comme acteur du développement. Les territoires multisitués ne sont pas ici des figures géographiques émergentes puisqu'ils sont à la fois "pré-modernes et postmodernes ", la dispersion, l'éclatement et la discontinuité étant depuis longtemps la base du fonctionnement spatial des sociétés maliennes. Mais leur configuration aujourd'hui se modifie sous l'effet à la fois de l'accélération des circulations migratoires et d'un nouveau maillage territorial lié à la décentralisation. Les territoires multisitués sont alors conçus, non pas seulement comme des territoires de pratiques et d'appartenance, mais aussi comme des territoires fonctionnels, organisationnels et institutionnels relevant de l'action légitime.

La contribution d'Elhadji Maman Moutari et de Frédéric Giraut va dans le même sens, en questionnant les territorialités que les corridors de transhumance au Sahel produisent. Instruments des politiques de soutien au pastoralisme, par nature «transterritoriaux", les corridors apparaissent comme des dispositifs réticulaires où sont mobilisés des lieux-ressources dispersés et complémentaires. Pour les auteurs, ces espaces multisitués font pourtant territoire en tant qu'ils impliquent des nouvelles formes de gestion, de régulation et de gouvernance politique sur lesquelles sont engagés à la fois les acteurs locaux, les collectivités des différents échelons territoriaux, ou encore les promoteurs et les acteurs de l'aide internationale (États, ONG, coopérations bilatérales). L'existence et la pérennité de ce territoire multisitué, participant d'un nouveau régionalisme africain, tiennent au fait, précisément, qu'ils s'articulent aux territoires plus classiques, de type aréolaire et contigu, que sont les terroirs, les départements ou encore les régions.

Ainsi, ces contributions, chacune à leur manière, dessinent les contours complexes de configurations sociospatiales où la multisituation peut faire territoire. La conceptualisation 
de ces formes territoriales, encore à ses prémisses, ouvre une voie prometteuse de réflexion qui ne demande qu'à être prolongée. Elle appelle aussi, certainement, à dépasser le seul champ de la géographie pour engager le dialogue avec d'autres champs disciplinaires des sciences sociales.

Geneviève Cortes Université Paul-Valéry Montpellier 3

Denis Pesche

CIRAD

Références

Antheaume B., Giraut F. (dir.)(2005). Le Territoire est mort, Vive les territoires! Paris: IRd Éditions, 384 p.

Arrault J.-B. (2005). « Du toponyme au concept? Usages et significations du terme archipel en géographie et dans les sciences sociales ». L'Espace géographique, t. 34, nº 4, p. 315-328.

Baudin G., Bonnin P. (dir.)(2009). Faire territoire. Paris: Recherches, 320 p.

Bauman Z. (1999). Le Coût humain de la mondialisation. Paris: Hachette Littérature, 204 p.

Brunet R., Ferras R., Théry H. (2009). Les Mots de la géographie. Dictionnaire critique. Paris: La Documentation française, coll. « Dynamiques du territoire », 518 p.

Capron G., Cortes G., Guétat-Bernard H. (2005). Liens et lieux de la mobilité. Ces autres territoires. Paris: Belin, coll. «Mappemonde», $344 \mathrm{p}$.

DıA H. (2010). "Les villages multisitués sénégalais face à la nouvelle configuration migratoire mondiale ». Hommes \& migrations, $n^{0}$ 1286-1287, p. 234-244.

Falzon M.A. (dir.)(2009). Multi-Sited Ethnography. Theory, Praxis and Locality in Contemporary Research. Farnham: Ashgate Publishing, $290 \mathrm{p}$.

HaEsbaert R. (2001). «Le mythe de la déterritorialisation ». Géographies et Cultures, n ${ }^{40}$. Paris: Éditions de l'Harmattan, p. 53-75.

Jessop B., Brenner N., Jones M. (2008). "Theorizing socio-spatial relations ». Environment and Planning D: Society and Space, vol. 26, p. 389-401.

Kaufmann J.-C. (2004). L'Invention de soi. Une théorie de l'identité. Paris: Armand Colin, 352 p.

LaPlantine F. (2005). Le Social et le Sensible. Introduction à une anthropologie modale. Paris: Téraèdre, coll. "L'anthropologue au coin de la rue », $220 \mathrm{p}$.

MARCUS G. (1995). «Ethnography in/of the world system: The emergence of multi-sited ethnography ». Annual Review of Anthropology, vol. 24, p. 95-117.

Pinedo-Vasquez M., Padoch C. (2009). « Urban and rural and in-between: Multisited households, mobility and resource management in the Amazon floodplain ». In AleXIADES M. (dir.), Mobility and Migration in Indigenous Amazonia. Oxford: Berghahn books, p. 86-96.

Romero C. (2010). "La cohésion territoriale et le développement local au défi des territorialités discontinues ». Management \& Avenir, 10, n 40, p 313-324.

SASSENS S. (2008). "Neither global nor national: novel assemblages of territory, authority and rights ». Ethics \& Global Politics, vol. 1, nº 1-2, p. 61-79.

Tarrius A. (2000). Les Nouveaux Cosmopolitismes. Mobilités, identités, territoires. La Tourd’Aigues: Éditions de l’Aube, coll. «Essai », 266 p.

VANIER M. (dir.)(2009). Territoires, Territorialité, Territorialisation. Controverses et perspectives. Rennes: Presses universitaires de Rennes, 228 p. 